PROCEEDINGS OF THE

AMERICAN MATHEMATICAL SOCIETY

Volume 125, Number 11, November 1997, Pages 3259-3265

S 0002-9939(97)04077-X

\title{
APPROXIMATION OF SINGULARITY SETS WITH ANALYTIC GRAPHS OVER THE BALL IN $\mathrm{C}^{2}$
}

\author{
MARSHALL A. WHITTLESEY \\ (Communicated by Eric Bedford)
}

\begin{abstract}
Let $h$ be a smooth function on the ball in $\mathbf{C}^{2}$ whose gradient has length less than or equal to 1 . We show that if $h$ is uniformly near an analytic function on every complex affine one-dimensional slice then it must be near some function analytic on the whole ball. We use this to show the following: a singularity set over the ball which is near the graph of a function $h$ with $|\nabla h| \leq 1$ must be near the graph of some analytic function over the ball.
\end{abstract}

Let $B_{2}$ be the open unit ball in $\mathbf{C}^{2}$, let $S=\partial B_{2}$ and let $K$ be a compact subset of $\bar{B}_{2} \times \mathbf{C}$. We say that $K$ is a singularity set if $\left(B_{2} \times \mathbf{C}\right) \backslash K$ is pseudoconvex. This implies that there exists an $f$ analytic on $\left(B_{2} \times \mathbf{C}\right) \backslash K$ which is singular at each point of the boundary of $K$ in $B_{2} \times \mathbf{C}$. If we let $\Delta$ denote the closed unit disk in $\mathbf{C}$ then we can make a similar definition of a singularity set in $($ int $\Delta) \times \mathbf{C}$. Singularity sets were studied as early as 1909 by Hartogs [3] and later by Oka [5] and Nishino [4]. One issue that has been studied is the question of whether such sets possess analytic structure, i.e., whether they contain analytic varieties. Wermer [9] and Słodkowski [8] showed that such an expectation is reasonable in general by proving a maximum modulus principle for singularity sets $K$ projecting onto $\Delta$; in particular, the following holds:

Proposition. If $\left(z_{0}, w_{0}\right) \in K$ then for every polynomial $Q$,

$$
\left|Q\left(z_{0}, w_{0}\right)\right| \leq \sup _{(z, w) \in K \cap\{|z|=1\}}|Q(z, w)| .
$$

(See (1) on p. 264 of [1].) In [1], Alexander and Wermer showed that a singularity set projecting onto the disk which is reasonably "thin" must be near an analytic graph. More precisely, they showed

Theorem of [1]. Let $\lambda \rightarrow a(\lambda)$ be a continuous function defined for $|\lambda| \leq 1$ with $|a(\lambda)| \leq 1$ for all $\lambda$. Fix $r>0$. Suppose that there exists a singularity set $X$ projecting onto $\Delta$ such that $X$ is contained in the tube $\{(\lambda, w)|| w-a(\lambda) \mid<r\}$. Then there exists an analytic function $\lambda \rightarrow f(\lambda)$ such that $|f(\lambda)-a(\lambda)| \leq 4 r$ for each $\lambda$ in the unit disk.

Received by the editors May 17, 1996.

1991 Mathematics Subject Classification. Primary 32E30, 32F15.

Key words and phrases. Singularity set, analytic structure.

This work is part of the author's Ph.D. thesis and was supported in part by the R. B. Lindsay Graduate Fellowship. The author would also like to express his appreciation for the guidance of his thesis advisor John Wermer.

(C) 1997 American Mathematical Society 
We shall make use of Alexander's and Wermer's technique in the proof of Theorem 2 .

If $h: \mathbf{C}^{2} \longrightarrow \mathbf{C}$ is a smooth function then $|\nabla h|$ shall denote the Euclidean length of the vector

$$
\left(\frac{\partial h}{\partial x_{1}}, \frac{\partial h}{\partial x_{2}}, \frac{\partial h}{\partial y_{1}}, \frac{\partial h}{\partial y_{2}}\right)
$$

where $\left(z_{1}, z_{2}\right)=\left(x_{1}+x_{2} i, y_{1}+y_{2} i\right)$. For $K$ a singularity set over the ball we shall prove the following.

Theorem 2. Let $h$ be a $C^{1}$ function in a neighborhood of the closed ball in $\mathbf{C}^{2}$ with $|\nabla h| \leq 1$ on $\bar{B}_{2}$. Suppose $K$ is a singularity set projecting onto $\bar{B}_{2}$ such that $K$ is contained in the tube

$$
T=\left\{(z, w) \epsilon \bar{B}_{2} \times \mathbf{C}|| w-h(z) \mid<\epsilon\right\} .
$$

Then there exists an analytic polynomial $F$ in $\mathbf{C}^{2}$ such that

$$
|F(z)-h(z)|<26 \sqrt{\epsilon} \text { on } \bar{B}_{2} .
$$

Suppose that $L$ is a complex affine subspace of $\mathbf{C}^{2}$ of complex dimension 1 which meets $B_{2}$ and let $p$ be the point on $L$ nearest to $(0,0)$. Then the points on $L$ which meet $S$ form a circle in $L$ with center $p$ and $L \cap \bar{B}_{2}$ is a disk embedded complex affinely in $\mathbf{C}^{2}$. We shall write $\Delta=L \cap \bar{B}_{2}$ and refer to $\Delta$ as a complex affine slice of $\bar{B}_{2}$. Our technique shall be to use the fact that $h$ is uniformly near an analytic function on every complex affine slice of $\bar{B}_{2}$ (from the results over the disk) and try to prove that $h$ is uniformly near an analytic function on $B_{2}$ as a function of two variables. We let $C\left(\bar{B}_{2}\right)$ denote the space of continuous complex-valued functions on $\bar{B}_{2}$ with supremum norm and $A\left(B_{2}\right)$ the subspace of functions which are analytic on $B_{2}$.

Theorem 1. Suppose $h$ is $C^{1}$ in a neighborhood of the closed ball in $\mathbf{C}^{2},|\nabla h| \leq 1$ on $\bar{B}_{2}$ and that given any complex affine slice $\Delta$ (a disk), there exists a polynomial $g_{\Delta}$ on $\Delta$ such that

$$
\left|h-g_{\Delta}\right|<\epsilon \text { on } \Delta \text {. }
$$

Then in $C\left(\bar{B}_{2}\right)$,

$$
\operatorname{dist}\left(h, A\left(B_{2}\right)\right)<13 \sqrt{\epsilon} .
$$

Proof. First we note that the theorem is trivial if $\epsilon \geq 1$, because then the fact that $|\nabla h| \leq 1$ means that $h$ is uniformly within 1 of the function which is constantly $h(0)$, and $1<13 \sqrt{1}$. Thus we assume that $\epsilon<1$.

We shall show that $C[h]$, the Cauchy integral of $h$, satisfies

$$
\operatorname{dist}\left(\left.C[h]\right|_{\Delta},\left.h\right|_{\Delta}\right)<\sqrt{5} \epsilon \text { in } L^{2}(\Delta)
$$

where $\Delta=$ a diametrical complex linear slice of $\bar{B}_{2}, \nu_{1}=$ normalized area measure on $\Delta$ and $L^{2}(\Delta)=$ the set of functions on $\Delta$ which are square integrable with respect to $\nu_{1}$. We shall also define $A^{2}(\Delta)=\left\{f\right.$ analytic in int $\left.\Delta \mid f \in L^{2}(\Delta)\right\}$. Suppose first that $\Delta=\bar{B}_{2} \cap\left\{\left(z_{1}, z_{2}\right) \mid z_{2}=0\right\}$. Let $\sigma=$ normalized volume measure 
on $S$. Using a technique used by Rudin in [6],

$$
\begin{aligned}
C[h]\left(z_{1}, 0\right) & =\int_{\partial B_{2}} \frac{h\left(\zeta_{1}, \zeta_{2}\right)}{\left(1-\left\langle z_{1}, \zeta_{1}\right\rangle\right)^{2}} d \sigma\left(\zeta_{1}, \zeta_{2}\right) \\
& =\int_{B_{1}} \frac{1}{\left(1-\left\langle z_{1}, \zeta_{1}\right\rangle\right)^{2}}\left(\frac{1}{2 \pi} \int_{-\pi}^{\pi} h\left(\zeta_{1}, \zeta_{2} e^{i \theta}\right) d \theta\right) d \nu_{1}\left(\zeta_{1}\right) .
\end{aligned}
$$

(See [6], pp. 15, 39.) For fixed $\zeta_{1}$ define $k\left(\zeta_{1}, \zeta_{2}\right)$ to be the harmonic extension of $h\left(\zeta_{1}, x\right),|x|=\sqrt{1-\left|\zeta_{1}\right|^{2}}$, to the region where $|x| \leq \sqrt{1-\left|\zeta_{1}\right|^{2}}$. Then the above equation becomes

$$
C[h]\left(z_{1}, 0\right)=\int_{B_{1}} \frac{k\left(\zeta_{1}, 0\right)}{\left(1-\left\langle z_{1}, \zeta_{1}\right\rangle\right)^{2}} d \nu_{1}\left(\zeta_{1}\right) .
$$

We check that $k$ is continuous when restricted to $\left\{z_{2}=0\right\}$. We have $k\left(z_{1}, 0\right)=$ $\frac{1}{2 \pi} \int_{-\pi}^{\pi} h\left(z_{1}, e^{i \theta} \sqrt{1-\left|z_{1}\right|^{2}}\right) d \theta$; for $z_{1}^{\prime}$ near $z_{1}, h\left(z_{1}^{\prime}, e^{i \theta} \sqrt{1-\left|z_{1}^{\prime}\right|^{2}}\right)$ is uniformly close to $h\left(z_{1}, e^{i \theta} \sqrt{1-\left|z_{1}\right|^{2}}\right)$ so $k\left(z_{1}^{\prime}, 0\right)$ is close to $k\left(z_{1}, 0\right)$.

Claim. For $\zeta_{1} \in \Delta$,

$$
\left|h\left(\zeta_{1}, 0\right)-k\left(\zeta_{1}, 0\right)\right|<2 \epsilon \text {. }
$$

Proof of Claim. From (1), we get that for fixed $\zeta_{1}$, there exists a polynomial $f_{\zeta_{1}}$ in $\zeta_{2}$ such that

$$
\left|h\left(\zeta_{1}, \zeta_{2}\right)-f_{\zeta_{1}}\left(\zeta_{2}\right)\right|<\epsilon \text { for }\left|\zeta_{2}\right| \leq \sqrt{1-\left|\zeta_{1}\right|^{2}}
$$

Thus

$$
\begin{aligned}
\left|h\left(\zeta_{1}, 0\right)-k\left(\zeta_{1}, 0\right)\right| & \leq\left|h\left(\zeta_{1}, 0\right)-f_{\zeta_{1}}(0)\right|+\left|f_{\zeta_{1}}(0)-k\left(\zeta_{1}, 0\right)\right| \\
& <\epsilon+\sup _{\left|\zeta_{2}\right|=\sqrt{1-\left|\zeta_{1}\right|^{2}}}\left|f_{\zeta_{1}}\left(\zeta_{2}\right)-k\left(\zeta_{1}, \zeta_{2}\right)\right| \\
& \left(\text { since } f_{\zeta_{1}} \text { and } k \text { are harmonic in } \zeta_{2}\right) \\
& =\epsilon+\sup _{\left|\zeta_{2}\right|=\sqrt{1-\left|\zeta_{1}\right|^{2}}}\left|f_{\zeta_{1}}\left(\zeta_{2}\right)-h\left(\zeta_{1}, \zeta_{2}\right)\right| \\
& <2 \epsilon,
\end{aligned}
$$

as claimed.

Let $\Pi: L^{2}(\Delta) \longrightarrow A^{2}(\Delta)$ be orthogonal projection. From what we know about the Bergman kernel, (4) is the orthogonal projection of $k$ to $A^{2}(\Delta)$, as a function of $z_{1}$. This means that $\Pi\left(\left.k\right|_{\Delta}\right)=\left.C[h]\right|_{\Delta}$. Then in $L^{2}(\Delta)$,

$$
\operatorname{dist}\left(\left.h\right|_{\Delta},\left.C[h]\right|_{\Delta}\right)^{2}=\operatorname{dist}\left(\left.h\right|_{\Delta}, \Pi\left(\left.h\right|_{\Delta}\right)\right)^{2}+\operatorname{dist}\left(\Pi\left(\left.h\right|_{\Delta}\right),\left.C[h]\right|_{\Delta}\right)^{2}
$$

by the Pythagorean theorem.

Since $\operatorname{dist}\left(\left.h\right|_{\Delta}, A(\Delta)\right)<\epsilon$ in $C(\Delta)$, $\operatorname{dist}\left(\left.h\right|_{\Delta}, A^{2}(\Delta)\right)<\epsilon$ in $L^{2}(\Delta)$, so we have $\operatorname{dist}\left(\left.h\right|_{\Delta}, \Pi\left(\left.h\right|_{\Delta}\right)\right)<\epsilon$ in $L^{2}(\Delta)$. Thus (6) is now

$$
\begin{gathered}
<\epsilon^{2}+\operatorname{dist}\left(\Pi\left(\left.h\right|_{\Delta}\right), \Pi\left(\left.k\right|_{\Delta}\right)\right)^{2} \\
\leq \epsilon^{2}+\operatorname{dist}\left(\left.h\right|_{\Delta},\left.k\right|_{\Delta}\right)^{2} \\
\leq \epsilon^{2}+(2 \epsilon)^{2}
\end{gathered}
$$

from (5)

$$
=5 \epsilon^{2} \text {. }
$$


Thus (3) holds for $\Delta=\bar{B}_{2} \cap\left\{\left(z_{1}, z_{2}\right) \mid z_{2}=0\right\}$.

Now if $\Delta$ is an arbitrary complex linear diametrical slice of $\bar{B}_{2}$, let $U$ be a unitary transformation such that $U\left(\left\{z_{2}=0\right\} \cap \bar{B}_{2}\right)=\Delta$. Then $h \circ U$ satisfies the original hypotheses of the theorem, so

$$
\begin{aligned}
& \operatorname{dist}\left(\left.h \circ U\right|_{\left\{z_{2}=0\right\}},\left.C[h \circ U]\right|_{\left\{z_{2}=0\right\}}\right)<\sqrt{5} \epsilon \text { in } L^{2}\left(B_{2} \cap\left\{z_{2}=0\right\}\right) \\
\Rightarrow & \operatorname{dist}\left(\left.h \circ U\right|_{\left\{z_{2}=0\right\}},\left.C[h] \circ U\right|_{\left\{z_{2}=0\right\}}\right)<\sqrt{5} \epsilon \text { in } L^{2}\left(B_{2} \cap\left\{z_{2}=0\right\}\right)
\end{aligned}
$$

(since the Cauchy integral operator commutes with unitary transformations). Since $U\left(\left\{z_{2}=0\right\} \cap \bar{B}_{2}\right)=\Delta$ and $U$ induces a natural isometry from $L^{2}(\Delta)$ to $L^{2}\left(B_{2} \cap\left\{z_{2}=0\right\}\right)$, we have (3).

Now let $f=C[h]$. Fix any diametrical slice $\Delta$. By (1) we can choose a polynomial $g_{\Delta} \epsilon A(\Delta)$ such that $\left|h-g_{\Delta}\right|<\epsilon$ on $\Delta$. Then $\operatorname{dist}\left(\left.h\right|_{\Delta}, g_{\Delta}\right)<\epsilon$ in $L^{2}(\Delta) \Rightarrow \operatorname{dist}\left(\left.f\right|_{\Delta}, g_{\Delta}\right) \leq \operatorname{dist}\left(f,\left.h\right|_{\Delta}\right)+\operatorname{dist}\left(\left.h\right|_{\Delta}, g_{\Delta}\right)$ in $L^{2}(\Delta)<\sqrt{5} \epsilon+\epsilon$ from $(3)$ $=(1+\sqrt{5}) \epsilon \equiv a \epsilon$ so that

$$
\operatorname{dist}\left(\left.f\right|_{\Delta}, g_{\Delta}\right)<a \epsilon \text { in } A^{2}(\Delta)
$$

Define

$$
\begin{aligned}
f^{r}\left(z e^{i \theta}\right) & =f\left(z r e^{i \theta}\right), z \in \operatorname{int} \Delta, \\
g_{\Delta}^{r}\left(z e^{i \theta}\right) & =g_{\Delta}\left(z r e^{i \theta}\right), z \in \Delta, \\
h^{r}\left(z e^{i \theta}\right) & =h\left(z r e^{i \theta}\right), z \in \Delta .
\end{aligned}
$$

Claim. For $r \leq 1-\sqrt{\epsilon}$,

$$
\left\|f^{r}-g_{\Delta}^{r}\right\|_{2} \leq a \epsilon^{\frac{3}{4}} \text { in } H^{2}(\Delta \cap S) .
$$

Proof of claim. Suppose not. Note that $\left\|f^{r}-g_{\Delta}^{r}\right\|_{2}$ is increasing in $r$ since $f^{r}$ and $g_{\Delta}^{r}$ are analytic. Thus

$$
\left\|f^{r}-g_{\Delta}^{r}\right\|_{H^{2}} \geq a \epsilon^{\frac{3}{4}} \text { for } r>1-\sqrt{\epsilon} .
$$

Using polar coordinates,

$$
\begin{aligned}
\int_{\Delta}\left|f-g_{\Delta}\right|^{2} d \nu_{1} & =2 \int_{0}^{1} r d r \frac{1}{2 \pi} \int_{-\pi}^{\pi}\left|f^{r}\left(e^{i \theta}\right)-g_{\Delta}^{r}\left(e^{i \theta}\right)\right|^{2} d \theta \\
& =2 \int_{0}^{1} r\left\|f^{r}-g_{\Delta}^{r}\right\|_{H^{2}}^{2} d r \\
& >2 \int_{1-\sqrt{\epsilon}}^{1} r\left\|f^{r}-g_{\Delta}^{r}\right\|_{H^{2}}^{2} d r \\
& \geq 2 \int_{1-\sqrt{\epsilon}}^{1} r a^{2} \epsilon^{\frac{3}{2}} d r, \text { from }(8) \\
& =2 a^{2} \epsilon^{\frac{3}{2}} \int_{1-\sqrt{\epsilon}}^{1} r d r \geq a^{2} \epsilon^{\frac{3}{2}}(2 \sqrt{\epsilon}-\epsilon) \\
& \geq a^{2} \epsilon^{\frac{3}{2}}(2 \sqrt{\epsilon}-\sqrt{\epsilon})=a^{2} \epsilon^{\frac{3}{2}} \sqrt{\epsilon}=a^{2} \epsilon^{2}
\end{aligned}
$$

(recall $\epsilon<1$ ) so $\left\|f-g_{\Delta}\right\|_{2}>a \epsilon$ in $A^{2}(\Delta)$, a contradiction of (7). Thus the claim (8) holds. This means that for $r \leq 1-\sqrt{\epsilon},\left\|f^{r}-h^{r}\right\|_{L^{2}(\Delta \cap S)} \leq\left\|f^{r}-g_{\Delta}^{r}\right\|_{L^{2}(\Delta \cap S)}$ 
$+\left\|g_{\Delta}^{r}-h^{r}\right\|_{L^{2}(\Delta \cap S)}<a \epsilon^{\frac{3}{4}}+\epsilon$ (from (1) and $\left.(8)\right) \leq(2+\sqrt{5}) \epsilon^{\frac{3}{4}}$ and we conclude

$$
\left\|f^{r}-h^{r}\right\|_{L^{2}(\Delta \cap S)} \leq(2+\sqrt{5}) \epsilon^{\frac{3}{4}} \text { for } r \leq 1-\sqrt{\epsilon} .
$$

Note that the conclusion holds for all diametrical slices $\Delta$.

Now define $F: B_{2} \longrightarrow \mathbf{C}$ by $F(z)=\int_{R} f\left(z e^{-i t}\right) \phi(t) d t$ where $\phi \epsilon C^{\infty}(\mathbf{R}), \phi \geq$ $0, \operatorname{spt} \phi \subset[-\sqrt{\epsilon}, \sqrt{\epsilon}], \int \phi d x=1$, and $\phi \leq \frac{1}{\sqrt{2 \epsilon}}$. Also define $H(z)=\int_{R} h\left(z e^{-i t}\right) \phi(t) d t$. Since $f$ and $\phi$ are smooth in $B_{2}, f$ analytic, $F$ is analytic in $B_{2}$ by differentiation under the integral sign. Then for $|z| \leq 1-\sqrt{\epsilon}$,

$$
\begin{aligned}
|F(z)-H(z)| & \leq\left|\int_{R}\left(f\left(z e^{-i t}\right)-h\left(z e^{-i t}\right)\right) \phi(t) d t\right| \\
& \leq \sqrt{\int_{-\sqrt{\epsilon}}^{\sqrt{\epsilon}}\left|f\left(z e^{-i t}\right)-h\left(z e^{-i t}\right)\right|^{2} d t} \sqrt{\int \phi(t)^{2} d t} \\
& \leq(\sqrt{2 \pi})(2+\sqrt{5}) \epsilon^{\frac{3}{4}} \sqrt{2 \sqrt{\epsilon} \sup _{t \in R}|\phi(t)|^{2}}, \text { from (9) } \\
& \leq(\sqrt{2 \pi})(2+\sqrt{5}) \epsilon^{\frac{3}{4}} \sqrt{2 \sqrt{\epsilon} \frac{1}{2 \epsilon}} \\
& \leq(\sqrt{2 \pi})(2+\sqrt{5}) \sqrt{\epsilon}
\end{aligned}
$$

and for $z \in B_{2}$,

$$
\begin{aligned}
|H(z)-h(z)| & =\left|\int_{R}\left(h\left(z e^{-i t}\right)-h(z)\right) \phi(t) d t\right| \\
& \leq \sup _{t \in \operatorname{spt\phi }}\left|h\left(z e^{-i t}\right)-h(z)\right|\|\phi\|_{1} \\
& \leq \sqrt{\epsilon}(\text { since }|\nabla h| \leq 1) .
\end{aligned}
$$

Thus for $|z| \leq 1-\sqrt{\epsilon}$,

$$
\begin{aligned}
|F(z)-h(z)| & \leq|F(z)-H(z)|+|H(z)-h(z)| \\
& \leq(\sqrt{2 \pi})(2+\sqrt{5}) \sqrt{\epsilon}+\sqrt{\epsilon}, \text { from }(10) \text { and }(11) \\
& <12 \sqrt{\epsilon}
\end{aligned}
$$

Lastly define $J: \bar{B}_{2} \longrightarrow \mathbf{C}$ by $J(z)=F(z(1-\sqrt{\epsilon}))$. Then $J \in A\left(B_{2}\right)$. For $z \in \bar{B}_{2}$,

$$
\begin{aligned}
|J(z)-h(z)| & =|F(z(1-\sqrt{\epsilon}))-h(z)| \\
& \leq|F(z(1-\sqrt{\epsilon}))-h(z(1-\sqrt{\epsilon}))|+|h(z(1-\sqrt{\epsilon}))-h(z)| \\
& <12 \sqrt{\epsilon}+\sqrt{\epsilon}, \text { from }(12) \text { and since }|\nabla h| \leq 1 \\
& \leq 13 \sqrt{\epsilon} .
\end{aligned}
$$

Thus $\operatorname{dist}\left(h, A\left(B_{2}\right)\right)<13 \sqrt{\epsilon}$ in $C\left(\bar{B}_{2}\right)$, which is (2).

We now prove Theorem 2, which we state again for the reader's convenience.

Theorem 2. Let h be a $C^{1}$ function in a neighborhood of the closed ball in $\mathbf{C}^{2}$ with $|\nabla h| \leq 1$ on $\bar{B}_{2}$. Suppose $K$ is a singularity set projecting onto $\bar{B}_{2}$ such that $K$ is contained in the tube

$$
T=\left\{(z, w) \epsilon \bar{B}_{2} \times \mathbf{C}|| w-h(z) \mid<\epsilon\right\} .
$$


Then there exists an analytic polynomial $F$ in $\mathbf{C}^{2}$ such that

$$
|F(z)-h(z)|<26 \sqrt{\epsilon} \text { on } \bar{B}_{2} \text {. }
$$

Proof. Our main goal is to show that on every complex affine slice $\Delta$ of $\bar{B}_{2}$, there exists a polynomial $g_{\Delta}$ such that

$$
\left|h-g_{\Delta}\right|<4 \epsilon .
$$

We can then apply Theorem 1 with (1) replaced by (15) and conclude (14) since $13 \sqrt{4}=26$. Let us now fix such a $\Delta$.

To prove (15), we can follow a method used by Alexander and Wermer in [2] to show that $\operatorname{dist}\left(\left.h\right|_{\Delta \cap S}, A(\Delta)\right)<2 \epsilon$. Let $P: \bar{B}_{2} \times \mathbf{C} \longrightarrow \bar{B}_{2}$ be projection and let $K^{\prime}$ be the set $\left\{(z, w)|| w-h(z) \mid \leq \epsilon, z \in \bar{B}_{2}\right\}$. We claim that: some element of the polynomial convex hull of $K^{\prime} \cap P^{-1}(\partial \Delta)$ lies over int $\Delta$. To see this, we first note that $K \cap P^{-1}($ int $\Delta)$ is a singularity set in $P^{-1}(\Delta)$ since the intersection of a pseudoconvex set with an affine subspace is pseudoconvex in that affine subspace. Then by the Proposition, the elements of $K \cap P^{-1}($ int $\Delta$ ) (which is nonempty by assumption) are in the polynomial hull of $K \cap P^{-1}(\partial \Delta)$. Since $K^{\prime} \supset K$, the claim holds. Then by Theorem 1 of [2], there exists $\phi \epsilon H^{\infty}(\Delta)$ such that $(z, \phi(z)) \in K^{\prime}$ for a.e. $z \in \Delta \cap S$. This means that $\|h-\phi\|_{\infty} \leq \epsilon<2 \epsilon$. Then we conclude that since $h$ is continuous, $\operatorname{dist}\left(\left.h\right|_{\Delta \cap S}, A(\Delta)\right)<2 \epsilon$. Choose a polynomial $g_{\Delta}$ on $\Delta$ such that

$$
\left|h-g_{\Delta}\right|<2 \epsilon \text { on } \Delta \cap S .
$$

Now consider the polynomial $w-g_{\Delta}$ on the set $K \cap(\Delta \times \mathbf{C})$. Let $\left(z_{0}, w_{0}\right) \in K \cap$ $(\Delta \times \mathbf{C})$. Since $\left(z_{0}, w_{0}\right)$ is in the polynomial hull of $K \cap P^{-1}(\partial \Delta)$,

$$
\left|w_{0}-g_{\Delta}\left(z_{0}, w_{0}\right)\right| \leq \sup _{(z, w) \in K, z \in \Delta \cap S}\left|w-g_{\Delta}(z, w)\right| .
$$

Then

$$
\begin{aligned}
\sup _{z \in \Delta}\left|h(z)-g_{\Delta}(z)\right| & \leq \sup _{(z, w) \in(K \cap(\Delta \times C))}|h(z)-w|+\sup _{(z, w) \in(K \cap(\Delta \times C))}\left|w-g_{\Delta}(z)\right| \\
& \leq \epsilon+\sup _{(z, w) \in K, z \in \Delta \cap S}\left|w-g_{\Delta}(z)\right|, \text { from }(13) \text { and }(17) \\
& \leq \epsilon+\sup _{(z, w) \in K, z \in \Delta \cap S}|w-h(z)|+\sup _{(z, w) \in K, z \in \Delta \cap S}\left|h(z)-g_{\Delta}(z)\right| \\
& <\epsilon+\epsilon+2 \epsilon, \text { from }(13) \text { and }(16) \\
& \leq 4 \epsilon .
\end{aligned}
$$

This shows that (15) holds, so the theorem is proven.

\section{REFERENCES}

1. Alexander, H., and J. Wermer, On the approximation of singularity sets by analytic varieties, Pacific J. Math. 104, No. 2 (1983), 263-267. MR 84e:32016

2. Alexander, H., and J. Wermer, Polynomial Hulls with Convex Fibers, Math. Ann. 271 (1985), 99-109. MR 86i:32025

3. Hartogs, F., Über die aus den singulären Stellen einer analytischen Funktion mehrerer Veränderlichen bestehenden Gebilde, Acta Math. 32 (1909), 57-79.

4. Nishino, T., Sur les ensembles pseudoconcaves, J. Math. Kyoto Univ. 1 (1962), 225-245. MR 26:5184

5. Oka, K., Notes sur les familles des fonctions analytiques multiform etc, Hiroshima Math. J., Series A 4 (1934), 93-98. 
6. Rudin, Walter, Function Theory in the Unit Ball of $\mathbf{C}^{n}$, Springer-Verlag, New York, 1980. MR 82i:32002

7. Słodkowski, Zbigniew, Analytic Set-Valued Functions and Spectra, Math. Ann. 256 (1981), 363-386. MR 83b:46070

8. Słodkowski, Zbigniew, Polynomial Hulls with Convex Sections and Interpolating Spaces, Proc. Amer. Math. Soc. 96, No. 2 (1986), 255-260. MR 87c:32023

9. Wermer, John, Maximum modulus algebras and singularity sets, Proc. Roy. Soc. Edinburgh Sect. A 86A (1980), 327-331. MR 82e:46071

Department of Mathematics, Brown University, Providence, Rhode Island 02912

E-mail address: mwhittle@math.brown.edu

Current address: Department of Mathematics, Texas A\&M University, College Station, Texas 77843-3368 